\title{
Research Article \\ Critical Study on Rotational Flow of a Fractional Oldroyd-B Fluid Induced by a Circular Cylinder
}

\author{
M. Kamran, ${ }^{1}$ M. Athar, ${ }^{2}$ and M. Imran ${ }^{2}$ \\ ${ }^{1}$ Department of Mathematics, COMSATS Institute of Information Technology, Wah Cantt 47040, Pakistan \\ ${ }^{2}$ Abdus Salam School of Mathematical Sciences, GC University, Lahore 54600, Pakistan \\ Correspondence should be addressed to M. Kamran, getkamran@gmail.com
}

Received 15 August 2011; Accepted 13 September 2011

Academic Editors: P. Minces and H. Zhou

Copyright (C) 2012 M. Kamran et al. This is an open access article distributed under the Creative Commons Attribution License, which permits unrestricted use, distribution, and reproduction in any medium, provided the original work is properly cited.

\begin{abstract}
We considered the unsteady flow of a fractional Oldroyd-B fluid through an infinite circular cylinder with the help of infinite Hankel and Laplace transforms. The motion of the fluid is produced by the cylinder that, at time $t=0^{+}$is subject to a time-dependent angular velocity. The established solutions have been presented under series form in terms of the generalized $G$ functions satisfy all imposed initial and boundary conditions. The corresponding solutions for ordinary Oldroyd-B, ordinary and fractional Maxwell, ordinary and fractional second-grade, and Newtonian fluids, performing the same motion, are acquired as limiting cases of general solutions. The keynote points regarding this work to mention are that (1) we extracted the expressions for velocity field and shear stress corresponding to the motion of fractional second-grade fluid as a limiting case of general solutions; (2) the expressions for velocity field and shear stress are in the most simplified form in contrast with the studies of Siddique and Sajid (2011), in which the expression for the velocity field involves the convolution product as well as the integral of the product of generalized $G$ functions. Finally, numerical results are presented graphically and discussed in order to reveal some physical aspects of obtained results.
\end{abstract}

\section{Introduction}

The motion of a fluid in cylindrical domains has applications in the food industry, oil exploitation, chemistry, and bioengineering [1], it being one of the most important problems of motion near translating or rotating bodies. The non-Newtonian fluids are now considered to play a more important and appropriate role in technological applications in comparison with Newtonian fluids. The first exact solutions corresponding to motions of non-Newtonian fluids in cylindrical domains seem to be those of Ting [2] for second-grade fluids, Srivastava [3] for Maxwell fluids, and Waters and King [4] for Oldroyd-B fluids. In the meantime a lot 
of papers regarding these motions have been published. However, the most of them deal with motion problems in which the velocity field is given on the boundary. To the best of our knowledge, the first exact solutions for motions of non-Newtonian fluids due to a shear stress on the boundary are those of Bandelli and Rajagopal [5] and Bandelli et al. [6] for second-grade fluids. Other similar solutions have been also obtained in [7-15].

Fractional calculus is a field of applied mathematics that deals with derivatives and integral of arbitrary orders [16, 17]. During the last decade fractional calculus has been applied to almost every field of science, engineering, and mathematics. A lot of applications of fractional calculus can be found in turbulence and fluid dynamics, plasma physics and controlled thermonuclear fusion, nonlinear control theory, nonlinear biological systems, and astrophysics [18-21] while its applications to polymer physics, biophysics, and thermodynamics can be found in [22]. The use of the method of fractional derivatives in the theory of viscoelasticity has the advantages that it affords possibilities for obtaining constitutive equations for elastic complex modulus of viscoelastic materials with only few experimentally determined parameters [23].

Our intention here is to establish exact solutions for the velocity field and the adequate shear stress corresponding to the unsteady flow of an incompressible Oldroyd-B fluid with fractional derivatives through an infinite circular cylinder. The motion of the fluid is produced by the cylinder, which, at time $t=0^{+}$, begins to rotate about its axis with a time-dependent angular velocity. The solutions that have been obtained, presented under series form in terms of the generalized $G$ functions, are established by means of the finite Hankel and Laplace transforms. The similar solutions for an ordinary Oldroyd-B, Maxwell with fractional derivatives, ordinary Maxwell fluids as well as those for the fractional and ordinary secondgrade fluids, can be obtained as limiting cases of general solutions for $\gamma, \beta \rightarrow 1 ; \lambda_{r} \rightarrow 0$ and $\beta \rightarrow 1 ; \lambda_{r} \rightarrow 0, \gamma$ and $\beta \rightarrow 1 ; \lambda \rightarrow 0$ and $\gamma \rightarrow 1 ; \lambda \rightarrow 0, \gamma$ and $\beta \rightarrow 1$, respectively. The solutions for a Newtonian fluid performing the same motion are obtained as limiting cases of the solutions for an Oldroyd-B fluid with fractional derivatives when $\lambda_{r} \rightarrow 0, \lambda \rightarrow 0$, and $\gamma, \beta \rightarrow 1$.

At the end with the help of Computer Algebra System (CAS) MATHCAD 2.0, we presented diagrams of velocity as well as of shear stress against different values of radius, time, and of the pertinent parameters. Also in one of the diagrams, we compared the velocity profiles and shear stresses corresponding to the motion of different fluid models, for the same value of time and of the common material parameters.

\section{Governing Equations}

The flows to be here considered have the velocity $\mathbf{v}$ and the extrastress $\mathbf{S}$ of the form [15]

$$
\mathbf{v}=\mathbf{v}(r, t)=w(r, t) \mathbf{e}_{\theta}, \quad \mathbf{S}=\mathbf{S}(r, t)
$$

where $\mathbf{e}_{\theta}$ is the unit vector in the $\theta$-direction of the cylindrical coordinates system $r, \theta$, and $z$. For such flows, the constraint of incompressibility is automatically satisfied. Furthermore, if initially the fluid is at rest, then

$$
\mathbf{v}(r, 0)=0, \quad \mathbf{S}(r, 0)=0 .
$$


The governing equations corresponding to such motions of Oldroyd-B fluid are given by [15]

$$
\begin{gathered}
\left(1+\lambda \frac{\partial}{\partial t}\right) \tau(r, t)=\mu\left(1+\lambda_{r} \frac{\partial}{\partial t}\right)\left(\frac{\partial}{\partial r}-\frac{1}{r}\right) w(r, t) \\
\left(1+\lambda \frac{\partial}{\partial t}\right) \frac{\partial w(r, t)}{\partial t}=v\left(1+\lambda_{r} \frac{\partial}{\partial t}\right)\left(\frac{\partial^{2}}{\partial r^{2}}+\frac{1}{r} \frac{\partial}{\partial r}-\frac{1}{r^{2}}\right) w(r, t)
\end{gathered}
$$

where $\mu$ is the dynamic viscosity, $v=\mu / \rho$ is the kinematic viscosity, with $\rho$ being the constant density of the fluid, $\lambda$ is the relaxation time, $\lambda_{r}$ is the retardation time, and $\tau(r, t)=S_{r \theta}(r, t)$ is the nontrivial shear stress.

The governing equations corresponding to an incompressible fractional Oldroyd-B fluid, performing the same motion, are obtained by replacing the inner time derivatives with respect to $t$ from (2.3), by the fractional differential operator given as [22-24]

$$
D_{t}^{\beta} f(t)= \begin{cases}\frac{1}{\Gamma(1-\beta)} \frac{d}{d t} \int_{0}^{t} \frac{f(\tau)}{(t-\tau)^{\beta}} d \tau, & 0 \leq \beta<1, \\ \frac{d}{d t} f(t), & \beta=1,\end{cases}
$$

where $\Gamma(\cdot)$ is the Gamma function defined by

$$
\Gamma(x)=\int_{0}^{\infty} t^{x-1} e^{-t} d t, \quad x>0
$$

Consequently, the governing equations to be used here are

$$
\begin{gathered}
\left(1+\lambda D_{t}^{r}\right) \tau(r, t)=\mu\left(1+\lambda_{r} D_{t}^{\beta}\right)\left(\frac{\partial}{\partial r}-\frac{1}{r}\right) w(r, t) \\
\left(1+\lambda D_{t}^{r}\right) \frac{\partial w(r, t)}{\partial t}=v\left(1+\lambda_{r} D_{t}^{\beta}\right)\left(\frac{\partial^{2}}{\partial r^{2}}+\frac{1}{r} \frac{\partial}{\partial r}-\frac{1}{r^{2}}\right) w(r, t) .
\end{gathered}
$$

We can easily observe that when $\gamma, \beta \rightarrow 1,(2.6)$ and (2.7) reduce to (2.3), because $D_{t}^{1} f=$ $d f / d t$. Furthermore, the new material constants $\lambda$ and $\lambda_{r}$ (although, for the simplicity, we keep the same notation) reduce to the previous ones for $\gamma, \beta \rightarrow 1$.

\section{Starting Flow through an Infinite Circular Cylinder}

Suppose that an incompressible fractional Oldroyd-B fluid is lying at rest in an infinite circular cylinder of radius $R(>0)$. At time $t=0^{+}$, the cylinder suddenly begins to rotate about its axis with an angular velocity $\Omega t^{2}$. Owing to the shear the inner fluid is gradually moved, 
with its velocity being of the form (2.1). The governing equations are given by (2.6) and (2.7), while the appropriate initial and boundary conditions are

$$
\begin{gathered}
w(r, 0)=\frac{\partial w(r, 0)}{\partial t}=0, \quad \tau(r, 0)=0 ; r \in[0, R], \\
w(R, t)=R \Omega t^{2}, \quad t \geq 0,
\end{gathered}
$$

where $\Omega$ is a constant.

The partial differential equations (2.6) and (2.7), also containing fractional derivatives, can be solved in principle by several methods; the integral transforms technique representing a systematic, efficient, and powerful tool. In the following we will use the Laplace transform to eliminate the time variable and the finite Hankel transform for the spatial variable. However, in order to avoid the burdensome calculations of residues and contour integrals, we will apply the discrete inverse Laplace transform method.

\subsection{Calculation of the Velocity Field}

Applying the Laplace transform to (2.7) and (3.2), we get

$$
\begin{gathered}
\left(q+\lambda q^{\gamma+1}\right) \bar{w}(r, q)= \\
v\left(1+\lambda_{r} q^{\beta}\right)\left(\frac{\partial^{2}}{\partial r^{2}}+\frac{1}{r} \frac{\partial}{\partial r}-\frac{1}{r^{2}}\right) \bar{w}(r, q), \\
\bar{w}(R, q)=\frac{2 R \Omega}{q^{3}}
\end{gathered}
$$

where $\bar{w}(r, q)$ and $\bar{w}(R, q)$ are the Laplace transforms of the functions $w(r, t)$ and $w(R, t)$, respectively. We will denote by [25]

$$
\bar{w}_{H}\left(r_{n}, q\right)=\int_{0}^{R} r \bar{w}(r, q) J_{1}\left(r r_{n}\right) d r
$$

the finite Hankel transform of the function $\bar{w}(r, q)$, and the inverse Hankel transform of $\bar{w}_{H}\left(r_{n}, q\right)$ is given by [25]

$$
\bar{w}(r, q)=\frac{2}{R^{2}} \sum_{n=1}^{\infty} \frac{J_{1}\left(r r_{n}\right)}{J_{2}^{2}\left(R r_{n}\right)} \bar{w}_{H}\left(r_{n}, q\right)
$$

where $r_{n}$ is the positive roots of the equation $J_{1}(R r)=0$ and $J_{p}(\cdot)$ is the Bessel function of the first kind of order $p$.

Multiplying now both sides of (3.3) by $r J_{1}\left(r r_{n}\right)$, integrating with respect to $r$ from 0 to $R$, and taking into account (3.4), (3.5), and the result which we can easily prove

$$
\int_{0}^{R} r\left(\frac{\partial^{2}}{\partial r^{2}}+\frac{1}{r} \frac{\partial}{\partial r}-\frac{1}{r^{2}}\right) \bar{w}(r, q) J_{1}\left(r r_{n}\right) d r=R r_{n} J_{2}\left(R r_{n}\right) \bar{w}(R, q)-r_{n}^{2} \bar{w}_{H}\left(r_{n}, q\right),
$$


we find that

$$
\bar{w}_{H}\left(r_{n}, q\right)=\left(v+v \lambda_{r} q^{\beta}\right) \Omega R^{2} r_{n} J_{2}\left(R r_{n}\right) \frac{2}{q^{3}\left(q+v r_{n}^{2}+\lambda q^{\gamma+1}+v r_{n}^{2} \lambda_{r} q^{\beta}\right)} .
$$

It can be also written in the suitable form

$$
\bar{w}_{H}\left(r_{n}, q\right)=\bar{w}_{1 H}\left(r_{n}, q\right)+\bar{w}_{2 H}\left(r_{n}, q\right)
$$

where

$$
\begin{gathered}
\bar{w}_{1 H}\left(r_{n}, q\right)=\frac{2 \Omega R^{2} J_{2}\left(R r_{n}\right)}{r_{n}} \frac{1}{q^{3}} \\
\bar{w}_{2 H}\left(r_{n}, q\right)=-\frac{2 \Omega R^{2} J_{2}\left(R r_{n}\right)}{r_{n}} \frac{1+\lambda q^{\gamma}}{q^{2}\left(q+v r_{n}^{2}+\lambda q^{\gamma+1}+v r_{n}^{2} \lambda_{r} q^{\beta}\right)} .
\end{gathered}
$$

Applying the inverse Hankel transform to (3.10) and using the known formula

$$
\int_{0}^{R} r^{2} J_{1}\left(r r_{n}\right) d r=\frac{R^{2}}{r_{n}} J_{2}\left(R r_{n}\right)
$$

we get

$$
\begin{gathered}
\bar{w}_{1}(r, q)=\frac{2 \Omega r}{q^{3}} \\
\bar{w}_{2}(r, q)=-4 \Omega \sum_{n=1}^{\infty} \frac{J_{1}\left(r r_{n}\right)}{r_{n} J_{2}\left(R r_{n}\right)} \frac{1+\lambda q^{\gamma}}{q^{2}\left(q+v r_{n}^{2}+\lambda q^{\gamma+1}+v r_{n}^{2} \lambda_{r} q^{\beta}\right)}
\end{gathered}
$$

Using the identity

$$
\frac{1}{\left(q+v r_{n}^{2}+\lambda q^{\gamma+1}+v r_{n}^{2} \lambda_{r} q^{\beta}\right)}=\frac{1}{\lambda} \sum_{k=0}^{\infty} \sum_{m=0}^{k} \frac{k !}{m !(k-m) !}\left(-\frac{v r_{n}^{2}}{\lambda}\right)^{k} \lambda_{r}^{m} \frac{q^{\beta m-k-1}}{\left(q^{\gamma}+\frac{1}{\lambda}\right)^{k+1}}
$$

(3.12b) can be written as

$$
\bar{w}_{2}(r, q)=-\frac{4 \Omega}{\lambda} \sum_{n=1}^{\infty} \frac{J_{1}\left(r r_{n}\right)}{r_{n} J_{2}\left(R r_{n}\right)} \sum_{k=0}^{\infty} \sum_{m=0}^{k} \frac{k !}{m !(k-m) !}\left(-\frac{v r_{n}^{2}}{\lambda}\right)^{k} \times \lambda_{r}^{m} \frac{\left(q^{\beta m-k-3}+\lambda q^{\gamma+\beta m-k-3}\right)}{\left(q^{\gamma}+1 / \lambda\right)^{k+1}}
$$


After taking the inverse Hankel transform of (3.9) and with the help of (3.12a) and (3.14), it leads to

$$
\bar{w}(r, q)=\frac{2 \Omega r}{q^{3}}-\frac{4 \Omega}{\lambda} \sum_{n=1}^{\infty} \frac{J_{1}\left(r r_{n}\right)}{r_{n} J_{2}\left(R r_{n}\right)} \sum_{k=0}^{\infty} \sum_{m=0}^{k} \frac{k !}{m !(k-m) !}\left(-\frac{v r_{n}^{2}}{\lambda}\right)^{k} \times \lambda_{r}^{m} \frac{\left(q^{\beta m-k-3}+\lambda q^{\gamma+\beta m-k-3}\right)}{\left(q^{\gamma}+1 / \lambda\right)^{k+1}}
$$

Now taking the inverse Laplace transform of (3.15), the velocity field $w(r, t)$ is given by

$$
\begin{aligned}
w(r, t)= & \Omega r t^{2}-\frac{4 \Omega}{\lambda} \sum_{n=1}^{\infty} \frac{J_{1}\left(r r_{n}\right)}{r_{n} J_{2}\left(R r_{n}\right)} \sum_{k=0}^{\infty} \sum_{m=0}^{k} \frac{k !}{m !(k-m) !}\left(-\frac{v r_{n}^{2}}{\lambda}\right)^{k} \lambda_{r}^{m} \\
& \times\left[G_{\gamma, \beta m-k-3, k+1}\left(-\lambda^{-1}, t\right)+\lambda G_{\gamma, \gamma+\beta m-k-3, k+1}\left(-\lambda^{-1}, t\right)\right],
\end{aligned}
$$

where the generalized function $G$ is defined by [26, Equations (97) and (101)]

$$
\begin{aligned}
G_{a, b, c}(d, t) & =\mathcal{L}^{-1}\left\{\frac{q^{b}}{\left(q^{a}-d\right)^{c}}\right\} \\
& =\sum_{k=0}^{\infty} \frac{d^{k} \Gamma(c+k)}{\Gamma(c) \Gamma(k+1)} \frac{t^{(c+k) a-b-1}}{\Gamma[(c+k) a-b]} ; \quad \operatorname{Re}(a c-b)>0,\left|\frac{d}{q^{a}}\right|<1 .
\end{aligned}
$$

\subsection{Calculation of the Shear Stress}

Applying the Laplace transform to (2.6), we find that

$$
\bar{\tau}(r, q)=\mu \frac{\left(1+\lambda_{r} q^{\beta}\right)}{\left(1+\lambda q^{r}\right)}\left(\frac{\partial}{\partial r}-\frac{1}{r}\right) \bar{w}(r, q)
$$

where, in view of (3.12a) and (3.12b), we have

$$
\begin{aligned}
\bar{w}(r, q) & =\bar{w}_{1}(r, q)+\bar{w}_{2}(r, q) \\
& =\frac{2 \Omega r}{q^{3}}-4 \Omega \sum_{n=1}^{\infty} \frac{J_{1}\left(r r_{n}\right)}{r_{n} J_{2}\left(R r_{n}\right)} \frac{1+\lambda q^{\gamma}}{q^{2}\left(q+v r_{n}^{2}+\lambda q^{\gamma+1}+v r_{n}^{2} \lambda_{r} q^{\beta}\right)}
\end{aligned}
$$

Now differentiating the last expression for $\bar{w}(r, q)$ partially with respect to $r$, we get

$$
\left(\frac{\partial}{\partial r}-\frac{1}{r}\right) \bar{w}(r, q)=-4 \Omega \sum_{n=1}^{\infty} \frac{J_{1}\left(r r_{n}\right)}{r_{n} J_{2}\left(R r_{n}\right)} \frac{1+\lambda q^{\gamma}}{q^{2}\left(q+v r_{n}^{2}+\lambda q^{\gamma+1}+v r_{n}^{2} \lambda_{r} q^{\beta}\right)}
$$


and introducing (3.20) into (3.18), we have

$$
\bar{\tau}(r, q)=4 \mu \Omega \sum_{n=1}^{\infty} \frac{J_{2}\left(r r_{n}\right)}{J_{2}\left(R r_{n}\right)} \frac{\left(1+\lambda_{r} q^{\beta}\right)}{q^{2}\left(q+v r_{n}^{2}+\lambda q^{\gamma+1}+v r_{n}^{2} \lambda_{r} q^{\beta}\right)} .
$$

In view of identity (3.13), (3.21) can be equivalently written as

$\bar{\tau}(r, q)=\frac{4 \mu \Omega}{\lambda} \sum_{n=1}^{\infty} \frac{J_{2}\left(r r_{n}\right)}{J_{2}\left(R r_{n}\right)} \sum_{k=0}^{\infty} \sum_{m=0}^{k} \frac{k !}{m !(k-m) !}\left(-\frac{v r_{n}^{2}}{\lambda}\right)^{k} \times \lambda_{r}^{m}\left[\frac{q^{\beta m-k-3}}{\left(q^{\gamma}+1 / \lambda\right)^{k+1}}+\lambda_{r} \frac{q^{\beta m+\beta-k-3}}{\left(q^{\gamma}+1 / \lambda\right)^{k+1}}\right]$.

Now taking the inverse Laplace transform of both sides of (3.22) and using (3.17), we find that

$$
\begin{aligned}
\tau(r, t)= & \frac{4 \mu \Omega}{\lambda} \sum_{n=1}^{\infty} \frac{J_{2}\left(r r_{n}\right)}{J_{2}\left(R r_{n}\right)} \sum_{k=0}^{\infty} \sum_{m=0}^{k} \frac{k !}{m !(k-m) !}\left(-\frac{v r_{n}^{2}}{\lambda}\right)^{k} \lambda_{r}^{m} \\
& \times\left[G_{\gamma, \beta m-k-3, k+1}\left(-\lambda^{-1}, t\right)+\lambda_{r} G_{\gamma, \beta m+\beta-k-3, k+1}\left(-\lambda^{-1}, t\right)\right] .
\end{aligned}
$$

\section{The Special Cases}

\subsection{Ordinary Oldroyd-B Fluid}

Making $\gamma, \beta \rightarrow 1$ into (3.16) and (3.23), we obtain the similar solutions for velocity field

$$
\begin{aligned}
w_{\mathrm{OO}}(r, t)= & \Omega r t^{2}-\frac{4 \Omega}{\lambda} \sum_{n=1}^{\infty} \frac{J_{1}\left(r r_{n}\right)}{r_{n} J_{2}\left(R r_{n}\right)} \sum_{k=0}^{\infty} \sum_{m=0}^{k} \frac{k !}{m !(k-m) !}\left(-\frac{v r_{n}^{2}}{\lambda}\right)^{k} \\
& \times \lambda_{r}^{m}\left[G_{1, m-k-3, k+1}\left(-\lambda^{-1}, t\right)+\lambda G_{1, m-k-2, k+1}\left(-\lambda^{-1}, t\right)\right]
\end{aligned}
$$

and shear stress

$$
\begin{aligned}
\tau_{\mathrm{OO}}(r, t)= & \frac{4 \mu \Omega}{\lambda} \sum_{n=1}^{\infty} \frac{J_{2}\left(r r_{n}\right)}{J_{2}\left(R r_{n}\right)} \sum_{k=0}^{\infty} \sum_{m=0}^{k} \frac{k !}{m !(k-m) !}\left(-\frac{v r_{n}^{2}}{\lambda}\right)^{k} \\
& \times \lambda_{r}^{m}\left[G_{1, m-k-3, k+1}\left(-\lambda^{-1}, t\right)+\lambda_{r} G_{1, m-k-2, k+1}\left(-\lambda^{-1}, t\right)\right],
\end{aligned}
$$

for an ordinary Oldroyd-B fluid performing the same motion. 


\subsection{Maxwell Fluid with Fractional Derivatives}

Making $\lambda_{r} \rightarrow 0, \beta \rightarrow 1$ into (3.16) and (3.23), we obtain velocity field

$$
\begin{aligned}
w_{\mathrm{FM}}(r, t)= & \Omega r t^{2}-\frac{4 \Omega}{\lambda} \sum_{n=1}^{\infty} \frac{J_{1}\left(r r_{n}\right)}{r_{n} J_{2}\left(R r_{n}\right)} \sum_{k=0}^{\infty}\left(-\frac{v r_{n}^{2}}{\lambda}\right)^{k} \\
& \times\left[G_{\gamma,-k-3, k+1}\left(-\lambda^{-1}, t\right)+\lambda G_{\gamma, \gamma-k-3, k+1}\left(-\lambda^{-1}, t\right)\right]
\end{aligned}
$$

and the associated shear stress

$$
\tau_{\mathrm{FM}}(r, t)=\frac{4 \mu \Omega}{\lambda} \sum_{n=1}^{\infty} \frac{J_{2}\left(r r_{n}\right)}{J_{2}\left(R r_{n}\right)} \sum_{k=0}^{\infty}\left(-\frac{v r_{n}^{2}}{\lambda}\right)^{k} G_{\gamma,-k-3, k+1}\left(-\lambda^{-1}, t\right),
$$

corresponding to a Maxwell fluid with fractional derivatives performing the same motion.

\subsection{Ordinary Maxwell Fluid}

Making $\gamma \rightarrow 1, \beta \rightarrow 1$, and $\lambda_{r} \rightarrow 0$ in (3.16) and (3.23), we get expressions for velocity field

$$
\begin{aligned}
w_{\mathrm{OM}}(r, t)= & \Omega r t^{2}-\frac{4 \Omega}{\lambda} \sum_{n=1}^{\infty} \frac{J_{1}\left(r r_{n}\right)}{r_{n} J_{2}\left(R r_{n}\right)} \sum_{k=0}^{\infty}\left(-\frac{v r_{n}^{2}}{\lambda}\right)^{k} \\
& \times\left[G_{1,-k-3, k+1}\left(-\lambda^{-1}, t\right)+\lambda G_{1,-k-2, k+1}\left(-\lambda^{-1}, t\right)\right]
\end{aligned}
$$

and the associated shear stress

$$
\tau_{\mathrm{OM}}(r, t)=\frac{4 \mu \Omega}{\lambda} \sum_{n=1}^{\infty} \frac{J_{2}\left(r r_{n}\right)}{J_{2}\left(R r_{n}\right)} \sum_{k=0}^{\infty}\left(-\frac{v r_{n}^{2}}{\lambda}\right)^{k} G_{1,-k-3, k+1}\left(-\lambda^{-1}, t\right),
$$

corresponding to an ordinary Maxwell fluid.

\subsection{Second-Grade Fluid with Fractional Derivatives}

Making $\lambda \rightarrow 0, \gamma \rightarrow 1$ into (3.16) and (3.23), and using (A1) and (A2), we obtain velocity field

$$
\begin{aligned}
w_{\mathrm{FS}}(r, t)= & \Omega r t^{2}-4 \Omega \sum_{n=1}^{\infty} \frac{J_{1}\left(r r_{n}\right)}{r_{n} J_{2}\left(R r_{n}\right)} \\
& \times \sum_{k=0}^{\infty}\left(-v r_{n}^{2}\right)^{k} G_{1-\beta,-\beta k-\beta-2, k+1}\left(-v \lambda_{r} r_{n}^{2}, t\right)
\end{aligned}
$$


and associated shear stress

$$
\begin{aligned}
\tau_{\mathrm{FS}}(r, t)= & 4 \mu \Omega \sum_{n=1}^{\infty} \frac{J_{2}\left(r r_{n}\right)}{J_{2}\left(R r_{n}\right)} \sum_{k=0}^{\infty}\left(-v r_{n}^{2}\right)^{k} \\
& \times\left[G_{1-\beta,-\beta k-\beta-2, k+1}\left(-v \lambda_{r} r_{n}^{2}, t\right)+\lambda_{r} G_{1-\beta,-\beta k-2, k+1}\left(-v \lambda_{r} r_{n}^{2}, t\right)\right],
\end{aligned}
$$

corresponding to a second-grade fluid with fractional derivatives performing the same motion. By taking $v \lambda_{r}=\alpha$ and $\alpha_{1}=\alpha \rho$ (the material constants for second grade fluid) into (4.7) and (4.8), we will get the similar solutions for fractional second-grade fluid as we get directly from the governing equations of fractional second-garde fluid as under

$$
\begin{gathered}
w_{\mathrm{FS}}(r, t)=\Omega r t^{2}-4 \Omega \sum_{n=1}^{\infty} \frac{J_{1}\left(r r_{n}\right)}{r_{n} J_{2}\left(R r_{n}\right)} \times \sum_{k=0}^{\infty}\left(-v r_{n}^{2}\right)^{k} G_{1-\beta,-\beta k-\beta-2, k+1}\left(-\alpha r_{n}^{2}, t\right), \\
\tau_{\mathrm{FS}}(r, t)=4 \Omega \sum_{n=1}^{\infty} \frac{J_{2}\left(r r_{n}\right)}{J_{2}\left(R r_{n}\right)} \sum_{k=0}^{\infty}\left(-v r_{n}^{2}\right)^{k} \times\left[\mu G_{1-\beta,-\beta k-\beta-2, k+1}\left(-\alpha r_{n}^{2}, t\right)+\alpha_{1} G_{1-\beta,-\beta k-2, k+1}\left(-\alpha r_{n}^{2}, t\right)\right] .
\end{gathered}
$$

\subsection{Ordinary Second-Grade Fluid}

Making $\beta \rightarrow 1$ into (4.9), we obtain velocity field

$$
w_{\mathrm{OS}}(r, t)=\Omega r t^{2}-4 \Omega \sum_{n=1}^{\infty} \frac{J_{1}\left(r r_{n}\right)}{r_{n} J_{2}\left(R r_{n}\right)} \sum_{k=0}^{\infty}\left(-v r_{n}^{2}\right)^{k} G_{0,-k-3, k+1}\left(-\alpha r_{n}^{2}, t\right)
$$

and the associated shear stress

$$
\begin{aligned}
\tau_{\mathrm{OS}}(r, t)= & 4 \Omega \sum_{n=1}^{\infty} \frac{J_{2}\left(r r_{n}\right)}{J_{2}\left(R r_{n}\right)} \sum_{k=0}^{\infty}\left(-v r_{n}^{2}\right)^{k} \\
& \times\left[\mu G_{0,-k-3, k+1}\left(-\alpha r_{n}^{2}, t\right)+\alpha_{1} G_{0,-k-2, k+1}\left(-\alpha r_{n}^{2}, t\right)\right],
\end{aligned}
$$

corresponding to an ordinary second-grade fluid. These solutions can also be simplified to give (see (A3)-(A4))

$$
\begin{gathered}
w_{\mathrm{OS}}(r, t)=\Omega r t^{2}-\frac{4 \Omega}{v} \sum_{n=1}^{\infty} \frac{J_{1}\left(r r_{n}\right)}{r_{n}^{3} J_{2}\left(R r_{n}\right)} \times\left[t+\frac{1+\alpha r_{n}^{2}}{v r_{n}^{2}}\left\{\exp \left(-\frac{v r_{n}^{2} t}{1+\alpha r_{n}^{2}}\right)-1\right\}\right], \\
\tau_{\mathrm{OS}}(r, t)=4 \rho \Omega \sum_{n=1}^{\infty} \frac{J_{2}\left(r r_{n}\right)}{r_{n}^{2} J_{2}\left(R r_{n}\right)}\left[t+\frac{1}{v r_{n}^{2}}\left\{\exp \left(-\frac{v r_{n}^{2} t}{1+\alpha r_{n}^{2}}\right)-1\right\}\right] .
\end{gathered}
$$




\subsection{Newtonian Fluid}

Making $\lambda_{r}, \lambda \rightarrow 0$ and $\gamma, \beta \rightarrow 1$ into (3.16) and (3.23) and using (A5) we get velocity field as

$$
w_{\mathrm{N}}(r, t)=\Omega r t^{2}-\frac{4 \Omega}{v} \sum_{n=1}^{\infty} \frac{J_{1}\left(r r_{n}\right)}{r_{n}^{3} J_{2}\left(R r_{n}\right)}\left[t+\frac{1}{v r_{n}^{2}}\left\{\exp \left(-v r_{n}^{2} t\right)-1\right\}\right]
$$

and the associated shear stress

$$
\tau_{\mathrm{N}}(r, t)=4 \rho \Omega \sum_{n=1}^{\infty} \frac{J_{2}\left(r r_{n}\right)}{r_{n}^{2} J_{2}\left(R r_{n}\right)}\left[t+\frac{1}{v r_{n}^{2}}\left\{\exp \left(-v r_{n}^{2} t\right)-1\right\}\right]
$$

corresponding to a Newtonian fluid performing the same motion. Of course, the Newtonian solutions (4.13) and (4.14) can be also obtained by making $\lambda \rightarrow 0$ into (4.5) and (4.6) and using (A5) or making $\alpha \rightarrow 0$ into (4.12).

\section{Conclusions}

The underlying idea of this research paper is to calculate exact solutions for velocity field and the adequate shear stress corresponding to the unsteady flow of an incompressible Oldroyd-B fluid with fractional derivatives through an infinite circular cylinder. The motion of the fluid is produced by the cylinder, which, at time $t=0^{+}$, begins to rotate about its axis subject to a time-dependent angular velocity. The solutions that have been obtained, presented under series form in terms of the generalized $G$ functions, are established by means of the finite Hankel and Laplace transforms. The similar solutions for an ordinary Oldroyd-B, Maxwell with fractional derivatives, ordinary Maxwell fluids as well as those for fractional and ordinary second-grade fluids, can be obtained as limiting cases of general solutions for $\gamma, \beta \rightarrow 1 ; \lambda_{r} \rightarrow 0$ and $\beta \rightarrow 1 ; \lambda_{r} \rightarrow 0, \gamma$ and $\beta \rightarrow 1 ; \lambda \rightarrow 0$ and $\gamma \rightarrow 1 ; \lambda \rightarrow 0, \gamma$ and $\beta \rightarrow 1$, respectively. The solutions for a Newtonian fluid performing the same motion are obtained as limiting cases of the solutions for an Oldroyd-B fluid with fractional derivatives when $\lambda_{r} \rightarrow 0, \lambda \rightarrow 0$, and $\gamma, \beta \rightarrow 1$.

\section{Numerical Results}

Finally, in order to reveal some relevant physical aspects of the obtained results, the diagrams of the velocity $w(r, t)$ as well as those of the shear stress $\tau(r, t)$, are depicted against $r$ for different values of time $t$ and of the pertinent parameters. Figures 1(a) and 1(b) clearly show that both velocity and the shear stress are increasing functions of $t$. They are also increasing functions of $r$, excepting $\tau(r, t)$ on a small interval near the boundary. The influence of the kinematic viscosity $v$ on the fluid motion is shown in Figures $2(\mathrm{a})$ and $2(\mathrm{~b})$, velocity $w(r, t)$ is an increasing function of $\mathcal{v}$, while shear stress $\tau(r, t)$ is a decreasing function of $\mathcal{v}$.

The influences of the relaxation time $\lambda$ and of the retardation time $\lambda_{r}$ on the velocity and shear stress are shown in Figures 3 and 4. The two parameters, as expected, have opposite 


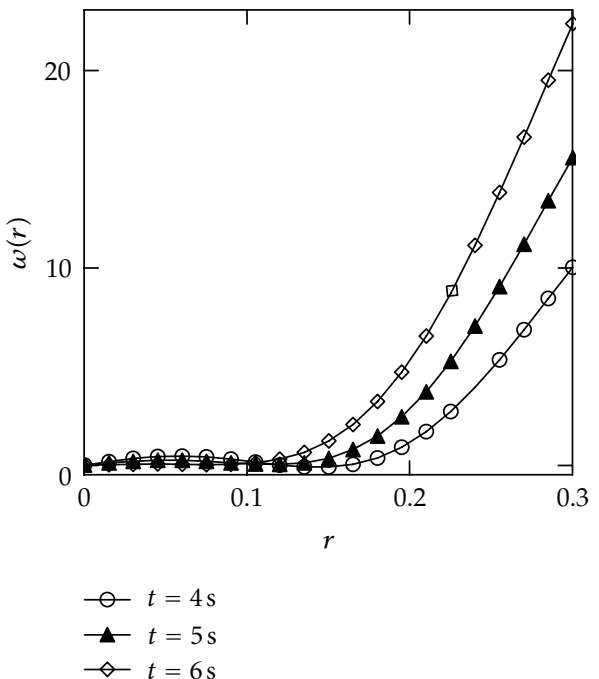

(a)

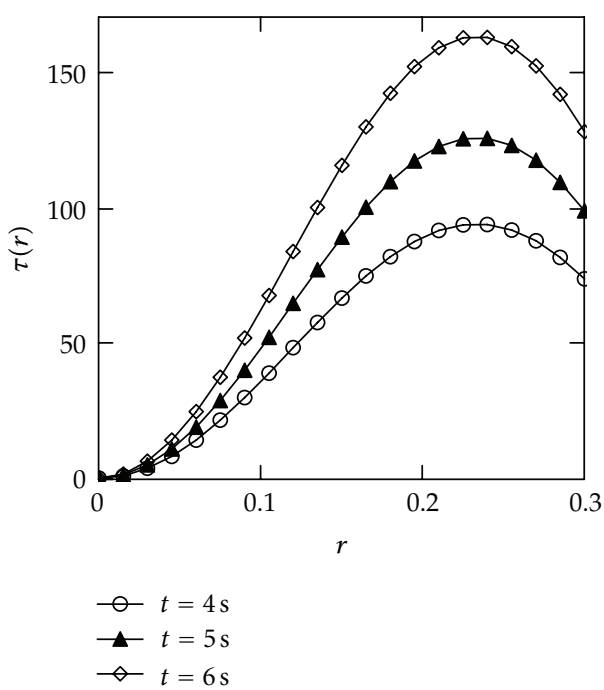

(b)

Figure 1: Profiles of the velocity $w(r, t)$ and shear stress $\tau(r, t)$ given by (3.16) and (3.23) for $R=0.3, \Omega=2$, $\lambda=3, \lambda_{r}=1, v=0.0031, \mu=2.96, \gamma=0.6, \beta=0.6$, and different values of $t$.

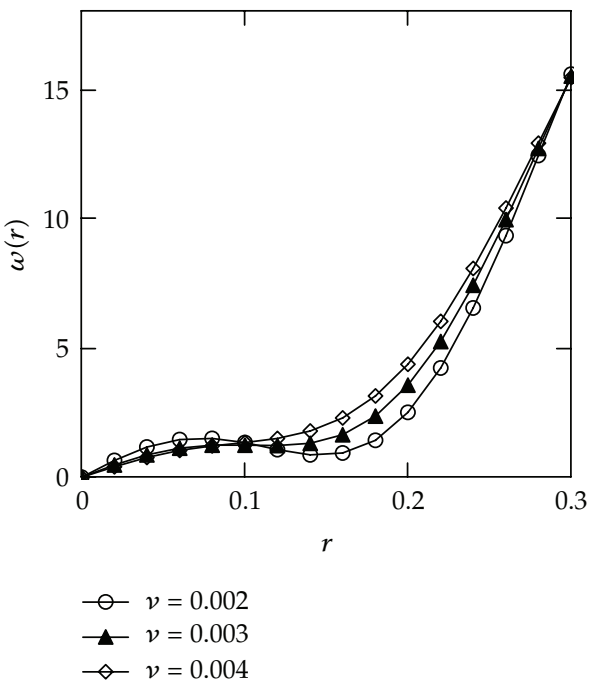

(a)

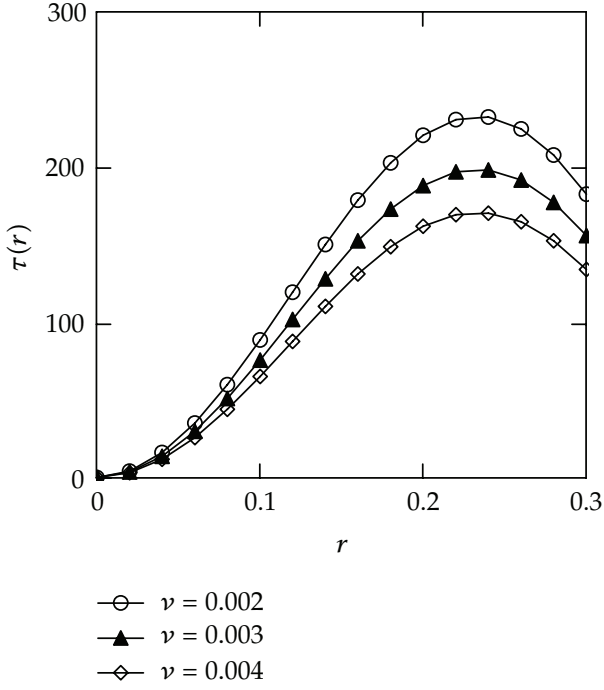

(b)

Figure 2: Profiles of the velocity $w(r, t)$ and shear stress $\tau(r, t)$ given by (3.16) and (3.23) for $t=5 \mathrm{~s}, R=0.3$, $\Omega=2, \lambda=3, \lambda_{r}=1, \mu=2.96, \gamma=0.7, \beta=0.3$, and different values of $\nu$.

effects on the fluid motion. More exactly, velocity and the shear stress are decreasing functions with respect to $\lambda$ and increasing ones with regards to $\lambda_{r}$. The influences of the fractional parameters $\gamma$ and $\beta$ on the fluid motion are presented in Figures 5 and 6 . Their effects are also opposite, but they are qualitatively the same as those of $\lambda_{r}$ and $\lambda$, respectively. More 


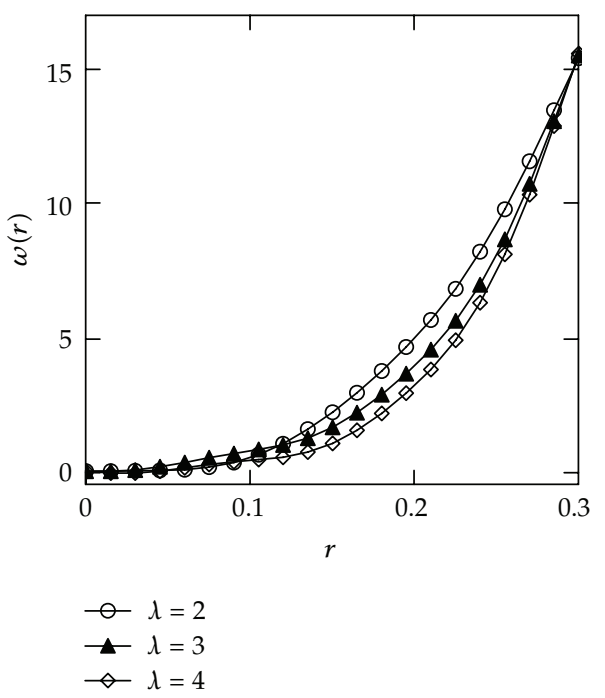

(a)

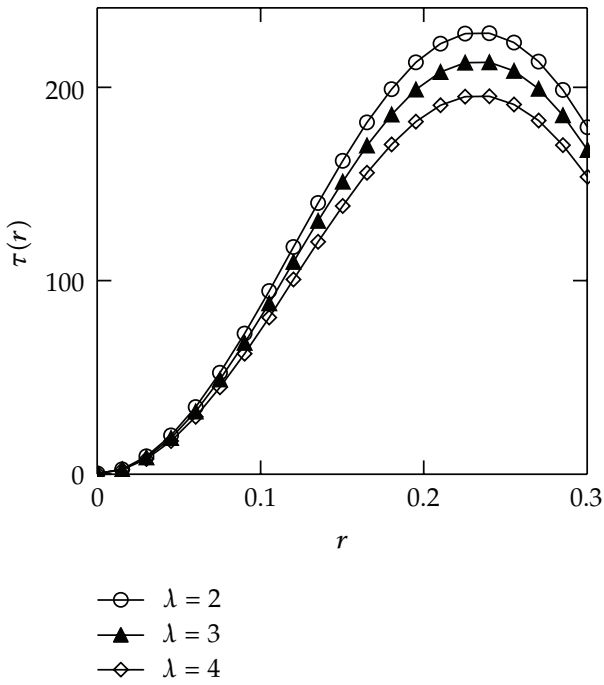

(b)

Figure 3: Profiles of the velocity $w(r, t)$ and shear stress $\tau(r, t)$ given by (3.16) and (3.23) for $t=5 \mathrm{~s}, R=0.3$, $\Omega=2, \lambda_{r}=1, v=0.003, \mu=2.96, \gamma=0.8, \beta=0.2$, and different values of $\lambda$.

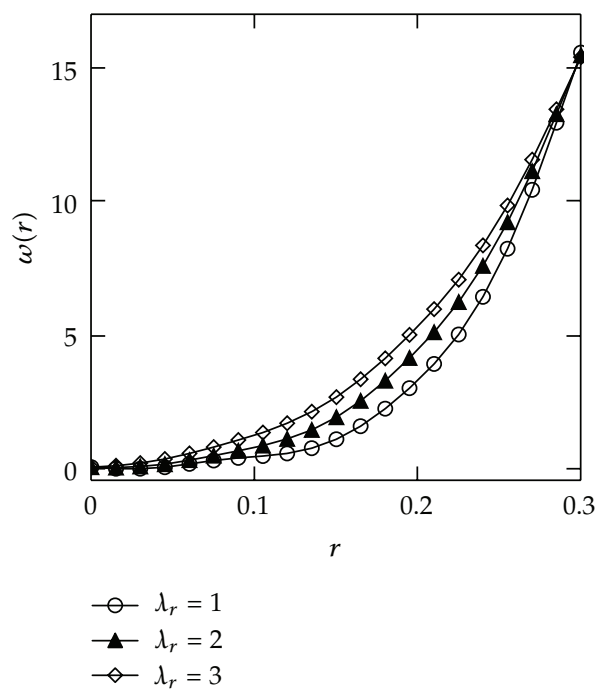

(a)

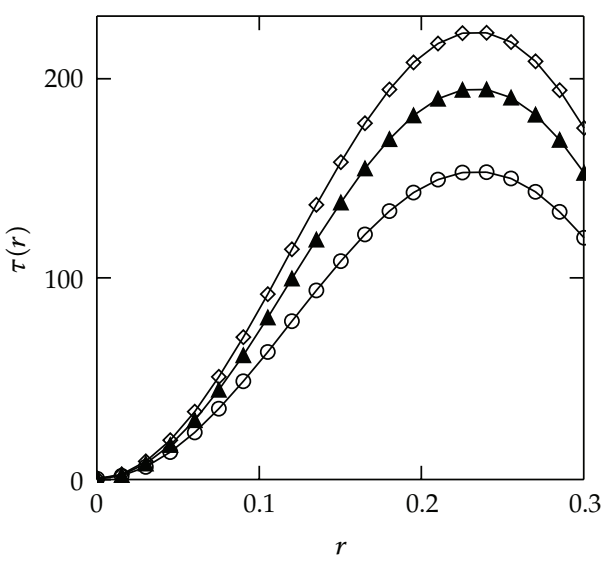

$$
\begin{aligned}
& \smile \lambda_{r}=1 \\
& \smile \lambda_{r}=2 \\
& \smile \lambda_{r}=3
\end{aligned}
$$

(b)

Figure 4: Profiles of the velocity $w(r, t)$ and shear stress $\tau(r, t)$ given by (3.16) and (3.23) for $t=5 \mathrm{~s}, R=0.3$, $\Omega=2, \lambda=4, v=0.003, \mu=2.96, \gamma=0.8, \beta=0.2$, and different values of $\lambda_{r}$.

exactly velocity $w(r, t)$ and the shear stress $\tau(r, t)$ are increasing functions with regards to $\gamma$ and decreasing with regards to $\beta$.

At the end, for comparison, the profiles of $w(r, t)$ corresponding to the motion of the seven models (Newtonian, ordinary second grade, fractional second grade, ordinary Maxwell, fractional Maxwell, ordinary Oldroyd-B, fractional Oldroyd-B) are together 


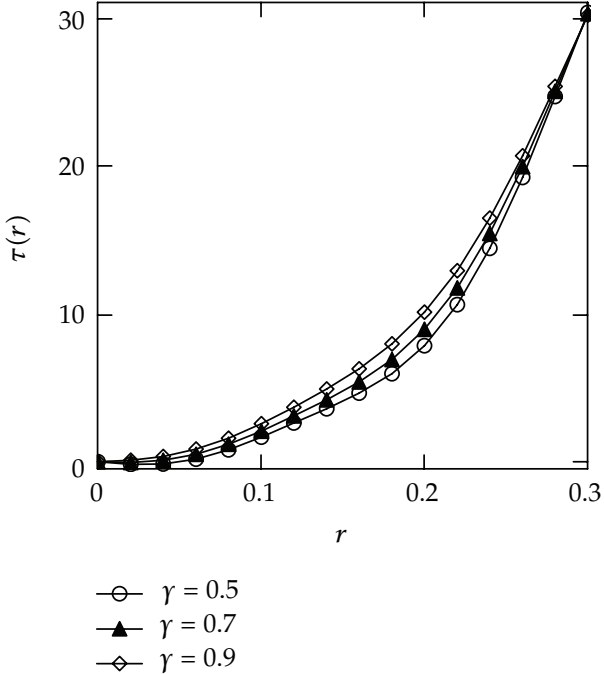

(a)

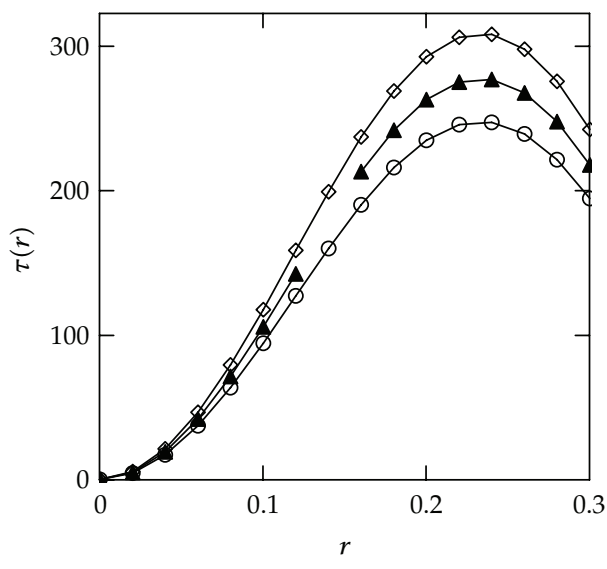

$\diamond \gamma=0.5$
$\multimap \gamma=0.7$
$\diamond \quad \gamma=0.9$

(b)

Figure 5: Profiles of the velocity $w(r, t)$ and shear stress $\tau(r, t)$ given by (3.16) and (3.23) for $t=7 \mathrm{~s}, R=0.3$, $\Omega=1, \lambda=5, \lambda_{r}=2, v=0.003, \mu=2.96, \beta=0.4$, and different values of $\gamma$.

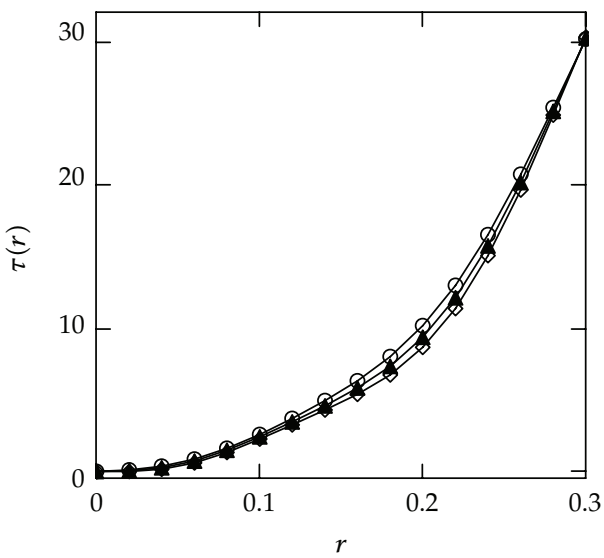

$\multimap \beta=0.1$
$\multimap \beta=0.4$
$\prec \beta=0.7$

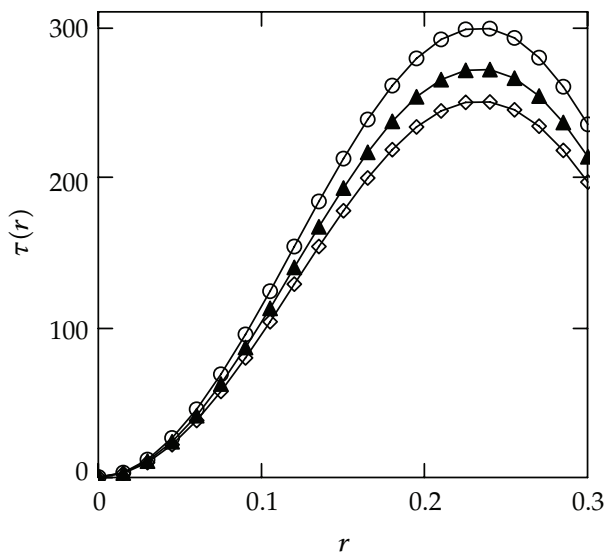

$\multimap \beta=0.1$

$\neg \beta=0.4$

$\multimap \beta=0.7$

(a)

(b)

Figure 6: Profiles of the velocity $w(r, t)$ and shear stress $\tau(r, t)$ given by (3.16) and (3.23) for $t=7 \mathrm{~s}, R=0.3$, $\Omega=2, \lambda=3, \lambda_{r}=1, v=0.0031, \mu=2.96, \gamma=0.7$, and different values of $\beta$.

depicted in Figure 7, for the same values of $t$ and of the common material parameters. The fractional Maxwell fluid, as it results from figure, is the slowest while the fractional secondgrade fluid is the swiftest on the whole flow domain. The units of the material constants are SI units within all figures, and the roots $r_{n}$ have been approximated by $(4 n+1) \pi /(4 R)$. 


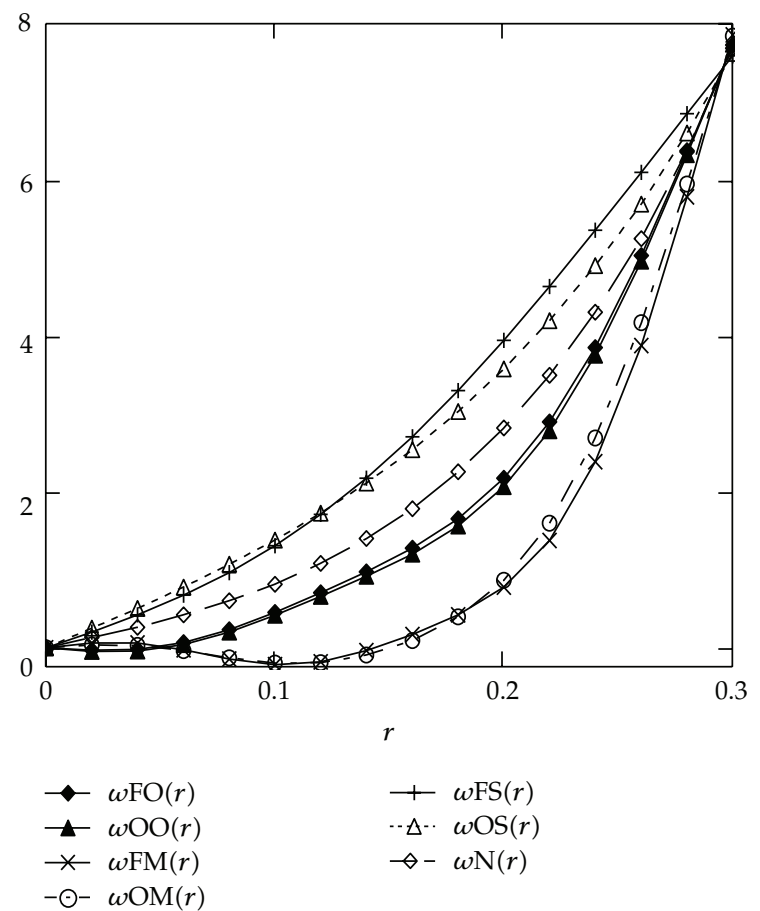

Figure 7: Profiles of the velocity $w(r, t)$ corresponding to the Newtonian, ordinary second grade, fractional second-grade, Maxwell, fractional Maxwell, Oldroyd-B and fractional Oldroyd-B fluids, for $t=5 \mathrm{~s}, R=0.3$, $\Omega=1, \lambda=4, \lambda_{r}=2, v=0.004, \mu=2, \gamma=0.7$, and $\beta=0.5$.

\section{Appendix}

One has

$$
\begin{gathered}
\sum_{k=0}^{\infty} \sum_{m=0}^{k} \frac{k !}{m !(k-m) !}\left(-v r_{n}^{2}\right)^{k} \lambda_{r}^{m} \frac{t^{-\beta m+k+2}}{\Gamma(-\beta m+k+3)}=\sum_{k=0}^{\infty}\left(-v r_{n}^{2}\right)^{k} G_{1-\beta,-\beta k-\beta-2, k+1}\left(-v \lambda_{r} r_{n}^{2}, t\right) \\
\sum_{k=0}^{\infty} \sum_{m=0}^{k} \frac{k !}{m !(k-m) !}\left(-v r_{n}^{2}\right)^{k} \lambda_{r}^{m} \frac{t^{-\beta m-\beta+k+2}}{\Gamma(-\beta m-\beta+k+3)}=\sum_{k=0}^{\infty}\left(-v r_{n}^{2}\right)^{k} G_{1-\beta,-\beta k-2, k+1}\left(-v \lambda_{r} r_{n}^{2}, t\right) \\
\sum_{k=0}^{\infty}\left(-v r_{n}^{2}\right)^{k} G_{0,-k-2, k+1}\left(-\alpha r_{n}^{2}, t\right)=\frac{1}{v r_{n}^{2}}\left\{1-\exp \left(-\frac{v r_{n}^{2} t}{1+\alpha r_{n}^{2}}\right)\right\} \\
\sum_{k=0}^{\infty}\left(-v r_{n}^{2}\right)^{k} G_{0,-k-3, k+1}\left(-\alpha r_{n}^{2}, t\right)=\frac{1}{v r_{n}^{2}}\left[t+\frac{1+\alpha r_{n}^{2}}{v r_{n}^{2}}\left\{\exp \left(-\frac{v r_{n}^{2} t}{1+\alpha r_{n}^{2}}\right)-1\right\}\right] \\
\lim _{\lambda \rightarrow 0} \frac{1}{\lambda^{k}} G_{a, b, k}\left(-\lambda^{-1}, t\right)=\frac{t^{-b-1}}{\Gamma(-b)}, \quad b<0
\end{gathered}
$$




\section{References}

[1] Y. Zhaosheng and L. Jianzhong, "Numerical research on the coherent structure in the viscoelastic second-order mixing layers," Applied Mathematics and Mechanics, vol. 19, no. 8, pp. 717-723, 1998.

[2] T. W. Ting, "Certain non-steady flows of second-order fluids," Archive For Rational Mechanics And Analysis, vol. 14, no. 1, pp. 1-26, 1963.

[3] P. H. Srivastava, "Non-steady helical flow of a visco-elastic liquid," Archives of Mechanics, vol. 18, pp. 145-150, 1966.

[4] N. D. Waters and M. J. King, "The unsteady flow of an elastico-viscous liquid in a straight pipe of circular cross section," Journal of Physics D, vol. 4, no. 2, article 304, pp. 204-211, 1971.

[5] R. Bandelli and K. R. Rajagopal, "Start-up flows of second grade fluids in domains with one finite dimension," International Journal of Non-Linear Mechanics, vol. 30, no. 6, pp. 817-839, 1995.

[6] R. Bandelli, K. R. Rajagopal, and G. P. Galdi, "On some unsteady motions of fluids of second grade," Archives of Mechanics, vol. 47, pp. 661-676, 1995.

[7] M. Imran, M. Athar, and M. Kamran, "On the unsteady rotational flow of a generalized Maxwell fluid through a circular cylinder," Archive of Applied Mechanics, vol. 81, pp. 1659-1666, 2011.

[8] M. Kamran, M. Athar, A. A. Zafar, and D. Vieru, "Axial Couette flow of a generalized second grade fluid due to a longitudinal time-dependent shear stress," Buletinul Institutului Politehnic Din Iasi, vol. 57, no. 61, 2011.

[9] M. Kamran, M. Imran, and M. Athar, "Exact solutions for the unsteady rotational flow of a generalized second grade fluid through a circular cylinder," Nonlinear Analysis: Modelling and Control, vol. 15, no. 4, pp. 437-444, 2010.

[10] M. Athar, M. Kamran, and C. Fetecau, "Taylor-Couette flow of a generalized second grade fluid due to a constant couple," Nonlinear Analysis: Modelling and Control, vol. 15, no. 1, pp. 3-13, 2010.

[11] M. E. Erdogan, "On unsteady motions of a second-order fluid over a plane wall," International Journal of Non-Linear Mechanics, vol. 38, no. 7, pp. 1045-1051, 2003.

[12] M. Imran, M. Kamran, M. Athar, and A. A. Zafar, "Taylor-Couette flow of a fractional second grade fluid in an annulus due to a time-dependent couple," Nonlinear Analysis: Modelling and Control, vol. 16, no. 1, pp. 47-58, 2011.

[13] C. Fetecau, C. Fetecau, and M. Imran, "Axial Couette flow of an Oldroyd-B fluid due to a timedependent shear stress," Mathematical Reports, vol. 11, no. 61, pp. 145-154, 2009.

[14] M. Khan, S. H. Ali, and C. Fetecau, "Exact solutions of accelerated flows for a Burgers' fluid Case-II," Zeitschrift fur Angewandte Mathematik und Physik, vol. 60, no. 4, pp. 701-722, 2009.

[15] C. Fetecau, M. Imran, C. Fetecau, and I. Burdujan, "Helical flow of an Oldroyd-B fluid due to a circular cylinder subject to time-dependent shear stresses," Zeitschrift fur Angewandte Mathematik und Physik, vol. 61, no. 5, pp. 959-969, 2010.

[16] A. A. Kilbas, H. M. Srivastava, and J. J. Trujillo, Theory and Applications of Fractional Differential Equations, Elsevier Science, New York, NY, USA, 2006.

[17] R. L. Magin, Fractional Calculus in Bioengineering, Begell House, Redding, Calif, USA, 2006.

[18] F. Mainardi, Yu. Luchko, and G. Pagnini, "The fundamental solution of the space time fractional diffusion equation," Fractional Calculus and Applied Analysis, vol. 4, no. 2, pp. 153-192, 2001.

[19] O. P. Agrawal, "Formulation of Euler-Lagrange equations for fractional variational problems," Journal of Mathematical Analysis and Applications, vol. 272, no. 1, pp. 368-379, 2002.

[20] V. E. Tarasov, "Fractional vector calculus and fractional Maxwell's equations," Annals of Physics, vol. 323, no. 11, pp. 2756-2778, 2008.

[21] M. A. E. Herzallah, A. M. A. El-Sayed, and D. Baleanu, "Perturbation for fractional-order evolution equation," Nonlinear Dynamics, vol. 62, no. 3, pp. 593-600, 2010.

[22] R. Hilfer, Applications of Fractional Calculus in Physics, World Scientific Press, Singapore, 2000.

[23] E. Soczkiewicz, "Application of fractional calculus in the theory of viscoelasticity," Molecular and Quantum Acoustics, vol. 23, pp. 397-404, 2002.

[24] I. Podlubny, Fractional Differential Equations, Academic Press, San Diego, Calif, USA, 1999.

[25] D. Tong and Y. Liu, "Exact solutions for the unsteady rotational flow of non-Newtonian fluid in an annular pipe," International Journal of Engineering Science, vol. 43, no. 3-4, pp. 281-289, 2005.

[26] C. F. Lorenzo and T. T. Hartley, "Generalized functions for the fractional calculus," NASA TP-1999209424/REV1, 1999. 


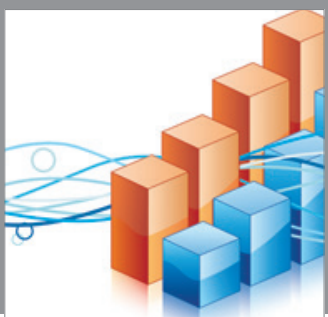

Advances in

Operations Research

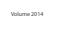

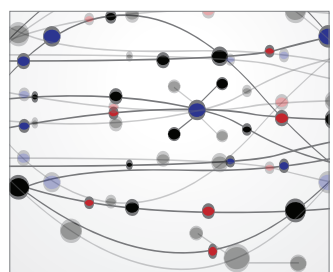

\section{The Scientific} World Journal
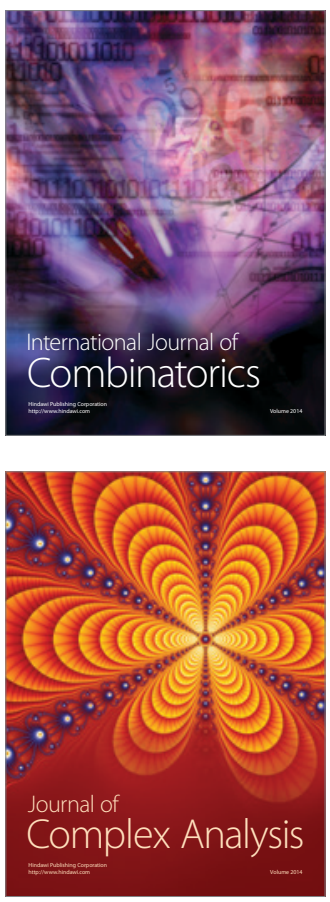

International Journal of

Mathematics and

Mathematical

Sciences
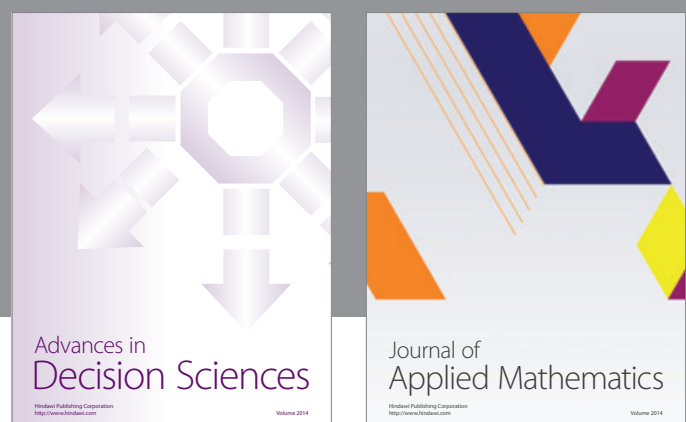

Journal of

Applied Mathematics
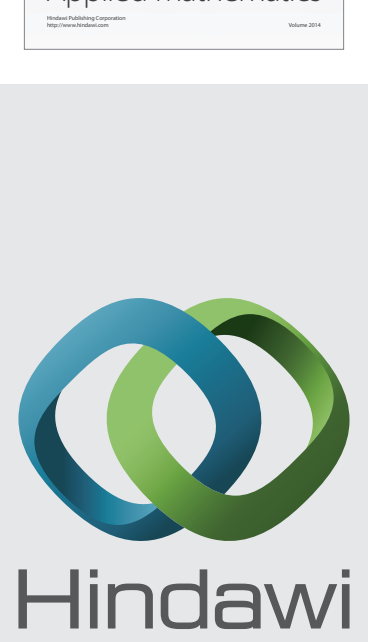

Submit your manuscripts at http://www.hindawi.com
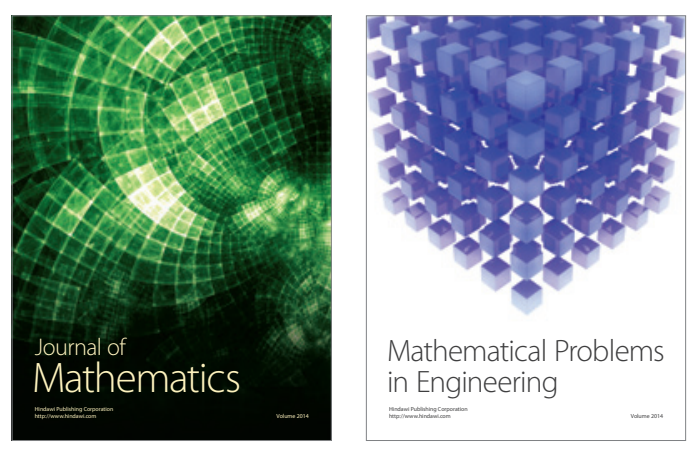

Mathematical Problems in Engineering
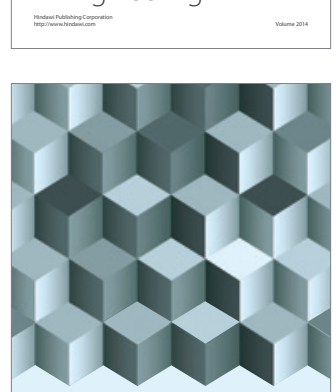

Journal of

Function Spaces
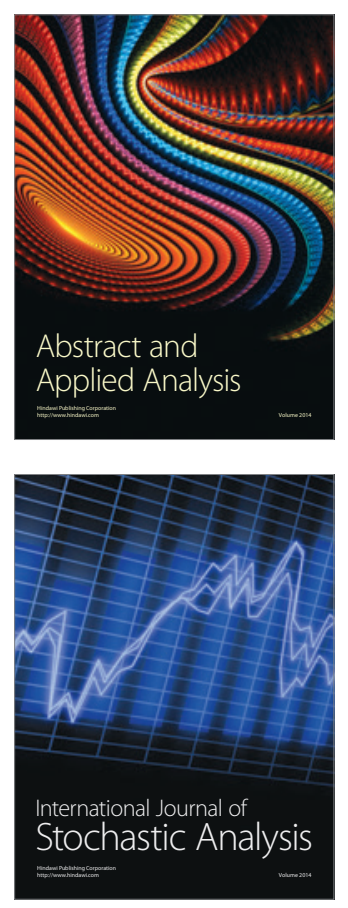

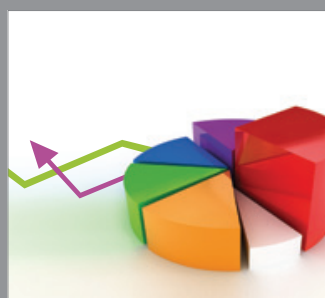

ournal of

Probability and Statistics

Promensencen
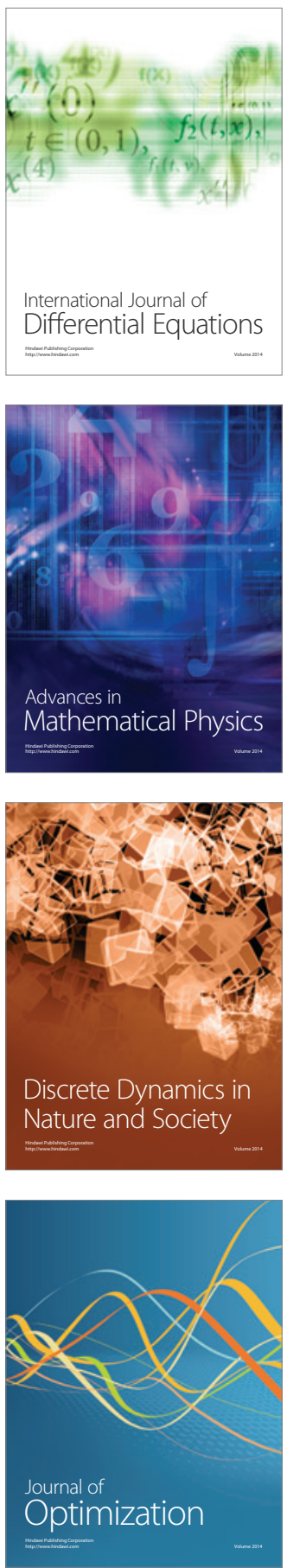